\title{
The efficacy of sodium fluorescein for visualizing biliary structures in a preclinical experience
}

\author{
Sungho KIM, Hyung Joon HAN, Tae-Jin SONG*
}

Department of Surgery, Korea University Ansan Hospital, Ansan, Korea

Introduction: Fluorescent imaging techniques have been introduced to promote real-time image-guided surgery and the technique has been widely adopted in hepatobiliary-pancreas surgery. The aim of this study is to validate the usefulness of blue light fluorescence imaging and fluorescein as a fluorophore in a mouse model.

Methods: Sodium fluorescein solution were administered intravenously to mouses, after laparotomy. The dose of solution was calculated based on the renal clearance of fluorescein. Whole abdominal organs were observed under blue light (wavelength of 440 to 490 nm) emitted from a LED curing light.

Results: After the solution was administered into the circulatory system of mouse, the bile duct under blue light emitted from the light source was well visualized.

Conclusions: This study established the preclinical basis of the blue light fluorescence imaging technique. The safety and feasibility of this technique should be demonstrated for human use. 